\title{
Percepciones sobre el abordaje de las Necesidades Educativas Especiales en la Práctica Profesional Virtual de Educación Física \\ Perceptions on addressing Special Educational Needs in the virtual professional practice of Physical Education
}

\author{
Franklin Castillo-Retamal, Alejandra Torres-Medina, Marilyn Herrera-Muñoz, César Faúndez-Casanova
}

Universidad Católica del Maule (Chile)

\begin{abstract}
Resumen. El objetivo de este trabajo es conocer el nivel de atención a los estudiantes que presentan necesidades educativas especiales (NEE) en los centros de Práctica de Síntesis Profesional (PSP) de Educación Física (EF) en tiempos de pandemia. La metodología utilizada responde a un análisis de tipo cuantitativo mediante un estudio exploratorio. La población de estudio determinada fue de 79 profesores en formación que desarrollaron su proceso de PSP durante el año 2020 y la muestra, de tipo no-probabilística por conveniencia, corresponde a 63 individuos. Los resultados indican que un $65,1 \%$ de los centros educativos demostró preocupación por lograr la inclusión de todos sus estudiantes en tiempos de pandemia, sin embargo, un $34,9 \%$ tuvo carencias en este aspecto, asimismo, un 53,9\% le otorga importancia a las NEE, por el contrario, y no menos importante, un 46,1\% indica que esto no fue así. En conclusión, se determinó que, si bien existió atención en muchos de los centros educativos estudiados, algunos de ellos todavía requieren mejorar en este aspecto para atender a sus estudiantes con NEE, evidenciando en estos últimos un nivel descendido de atención.
\end{abstract}

Palabras clave: Educación Física; formación docente; pandemia; inclusión; escuela.

\begin{abstract}
The objective of this work is to know the level of attention of the students who present SEN in the Professional Synthesis Practice centers (PSP) in times of pandemic. The methodology used responds to a quantitative analysis through an exploratory study. The study population determined was 79 students who developed their professional synthesis practice process during 2020 and the non-probabilistic sample for convenience corresponds to 63 individuals. The results indicate that $65.1 \%$ of the educational centers showed concern about achieving the inclusion of all their students in times of pandemic, however $34.9 \%$ had deficiencies in this aspect, likewise, 53.9\% grant it importance to SEN, on the contrary and no less important, $46.1 \%$ indicate that this was not the case. In conclusion, it was determined that although there was attention in many of the educational centers studied, some of them still require improvement in this aspect to serve their students with SEN, appreciating a decreased level in the latter.
\end{abstract}

Key words: Physical Education; teacher training; pandemic; inclusion; school.

\section{Introducción}

En las últimas décadas, la necesidad de mejorar la calidad y equidad de la educación escolar ha sido un elemento relevante en la discusión pública. Con ello, sin duda, las temáticas de inclusión y diversidad no han estado exentas de reflexión, esto según lo señalado por la Agencia de Calidad de la Educación (2018). En Chile, Molina (2015) indica que se han implementado reformas educativas destinadas principalmente a mejorar la calidad, equidad y eficiencia de los sistemas educativos para mejorar los aprendizajes elementales de los estudiantes, incluidos aquellos que presentan NEE. Al respecto, el Ministerio de Educación de Chile (MINEDUC) señala que

Fecha recepción: 21-01-21. Fecha de aceptación: 25-05-21

Franklin Castillo-Retamal

fcastillo@ucm.cl
«La reforma educacional actual, implica una serie de cambios sistémicos que han apuntado a construir un sistema educacional inclusivo, que efectivamente promueva una educación de calidad integral, donde la valoración de la diversidad, así como otros aspectos valóricos y de educación ciudadana, vuelvan a constituir ámbitos y temáticas fundamentales en la formación y el aprendizaje de los estudiantes» (MINEDUC, 2016, p.7).

Hoy en día, según el informe de la Agencia de Calidad de Educación (2018), el sistema escolar chileno enfrenta importantes desafíos. Si bien el país ha tenido grandes avances, un porcentaje importante de nuestros estudiantes no está alcanzando los aprendizajes esperados para su edad, siendo parte también de este grupo alumnos que presentan NEE, en donde es posible observar que cada vez es mayor la cantidad de estudiantes que ingresan a la educación regular o especial, presentando NEE asociadas a un déficit o a una condición de discapacidad (Castillo et al., 2021). De lo anterior, se- 
gún la base de datos del MINEDUC, en nuestro país el año 2018 hubo 183.373 alumnos con NEE matriculados, número que representó un 5,12\% de la matrícula total nacional (Holz, 2018). En este sentido Jiménez y Montecinos (2018, p.3), indican que «en este escenario, uno de los principales desafíos del sistema educativo chileno es resolver las profundas desigualdades de aprendizaje y participación que afecta a una parte significativa de sus estudiantes».

Nuestra sociedad ha experimentado un proceso continuo de diversos cambios, entre los que podemos reconocer, indiscutiblemente, aquellos que se han producido en el ámbito de la educación. Dichos cambios han surgido en su mayoría, según Navarrete (2019) y Castillo et al. (2015), de la diversidad y diferencia. La primera, se debe considerar como característica única de la naturaleza humana, debiendo considerar la totalidad de la expresión del sujeto, mientras que la segunda, se debe traducir como una condición individual de cada ser humano, que es necesario atender de forma permanente.

Dentro de esta diversidad se encuentran los estudiantes que presentan algún tipo de NEE, identificados por el MINEDUC (2015) y explicitados en el art. 23 de la Ley General de Educación (2009), como «aquel que precisa ayudas y recursos adicionales, ya sea humanos, materiales o pedagógicos, para conducir su proceso de desarrollo y aprendizaje, y contribuir al logro de los fines de la educación» (p.15), es decir, requiere de una educación que brinde al estudiante la mayor posibilidad de oportunidades por medio de la utilización de diversos recursos adicionales, entre los que se encuentran los humanos, profesionales y docentes que a diario forman parte del contexto del educando. En otras palabras, para lograr atender las NEE de aquel estudiante que las presenta, el docente debe ser capaz de aplicar estrategias de enseñanza adecuadas y recursos metodológicos para dar respuesta a un contexto educativo heterogéneo.

De lo anterior y tomando en cuenta el gran desafío por disminuir las barreras de desigualdad en la educación de nuestro país, es que nace un nuevo desafío a mayor escala que nos afecta tanto a nivel país como a nivel mundial, una nueva realidad a la que nunca nos habíamos enfrentado, una pandemia que ha sido provocada por el denominado COVID-19, que es «una enfermedad infecciosa nueva que puede presentar manifestaciones clínicas graves, incluyendo la muerte, presente ya en 124 países» (Emanuel et. al, 2020, p. 2049).

Dado este contexto de pandemia, la educación en nuestro país se vio en la obligación de adecuarse a esta nueva realidad y utilizar la virtualidad como medio para llevar a cabo las clases a lo largo de todo el territorio nacional, luego de suspender las clases presenciales con el propósito de no exponer a los estudiantes y profesionales de la educación a un posible contagio, un método que ha impactado de forma directa en el proceso de enseñanza-aprendizaje de todos los estudiantes, puesto que ha sido necesario adaptarse de manera significativa luego del cierre de los establecimientos educacionales (Álvarez \& Figares, 2020; Flores-Ferro et al., 2021; Almonacid et al., 2021).

A partir de los antecedentes presentados, el desarrollo del texto estará orientado a la descripción de las medidas adoptadas por el Gobierno de Chile en el ámbito educativo, cuyo propósito es continuar con el proceso de enseñanza aprendizaje a través de la modalidad virtual, siendo específicamente nuestro punto de enfoque las clases de Educación Física y Salud impartidas por profesores en formación en etapa de PSP y, cómo por medio de ellas, se atienden las NEE de los estudiantes.

\section{Educación Física y necesidades educativas es- peciales desde la virtualidad}

La pandemia generada por el COVID-19 se ha transformado en uno de los desafíos más grandes que ha tenido que enfrentar la población mundial durante el presente siglo, Arriagada (2020) indica que a causa de esta alerta sanitaria mundial «el gobierno de Chile, según recomendaciones de la OMS (Organización Mundial de la Salud) y especialistas locales, tomó como una de las medidas sanitarias preventivas y de contingencia, el cierre de todos los establecimientos del sistema escolar»(p.1), a través de la Resolución Exenta n 322 publicada por el Ministerio de Salud (MINSAL, 2020), que resuelve la suspensión presencial de clases en los establecimientos educacionales del país con la intención de frenar la propagación de esta enfermedad, convirtiendo el distanciamiento físico en la medida no farmacéutica de mayor masividad.

En este contexto, la provisión de educación a distancia pasó a adquirir una gran relevancia para la sociedad en su conjunto, en donde el MINEDUC ha tomado una serie de medidas para afrontar esta nueva realidad, la que ha afectado a más de 3 millones de estudiantes de educación parvularia, primaria y secundaria. A raíz de lo anterior, el MINEDUC a través de la Resolución Exenta $n^{\circ} 2765$, con la responsabilidad de asegurar el derecho a la educación, ha impulsado una Priorización Curricular (PC) que aprueba las orientaciones para su implementación, cuyo propósito es responder a los pro- 
blemas que surjan debido a la paralización de clases presenciales y, en consecuencia, de la reducción de semanas lectivas, convirtiéndose en una herramienta de apoyo curricular para las escuelas que permita enfrentar y minimizar las consecuencias adversas que han emergido.

Esta priorización, según el MINEDUC (2020), responde a tres principios básicos: seguridad, flexibilidad y equidad, formulada para ser implementada durante los años 2020 y 2021 como espacios de recuperación y reforzamiento de aprendizajes fundamentales en los cuales, dependiendo del contexto, se transite desde la PC hacia el currículum vigente, el que se pretende retomar en marzo del año 2022.

En el caso de las clases de Educación Física y Salud y la atención a las NEE también han debido adaptarse a la virtualidad, en donde según indican Murillo \& Duk (2020) y Álvarez \& Figares (2020), los niños, niñas y adolescentes en situación de discapacidad, han sido el colectivo que más ha sufrido durante esta pandemia mundial, por lo tanto, es de esperar que esto tenga consecuencias a la hora de volver a la actividad escolar presencial.

«En este proceso de transición han tenido un papel fundamental las nuevas tecnologías, la colaboración de los padres y la capacidad de adaptación de los profesionales de la educación. Las nuevas tecnologías y medios de comunicación han servido, por ejemplo, para que alumnos y profesores puedan intercambiar material didáctico en diferentes formatos, interactuar en aulas virtuales y que se mantenga un seguimiento o evaluación de las diferentes actividades» (Álvarez \& Figares, 2020, p.2).

De lo anterior, los mismos autores señalan que en esta nueva modalidad de llevar a cabo las clases, existen tres grandes retos que las escuelas deben afrontar: «dudas sobre la efectividad de las intervenciones educativas sobre alumnos con NEE en un medio virtual, dificultades de comunicación con las familias y los alumnos y, por último, el desgaste emocional asociado al trabajo en este nuevo contexto educativo» (Álvarez-Hevia \& Figares, 2020,p.3).

Por otro lado, es importante considerar que en relación a los estudiantes con NEE, «los roles y responsabilidades de sus familias son más complejos, dada la especificidad de los apoyos que requieren con algún tipo de atención particular: no todos los adultos responsables tienen las herramientas y técnicas requeridas» (Educación 2020, 2020, p.5). Sumado a esto, una variable relevante a considerar es que la comunicación oral ha sido reemplazada por la comunicación escrita, puesto que la herramienta que más se utiliza como medio para la entrega de información y materiales para los estudiantes con NEE es el correo electrónico, lo que provoca cierta incomodidad en muchas familias, «ya que en ocasiones hay que explicar en varios correos los objetivos y las tareas que se plantean, con el consiguiente consumo de tiempo que ello implica y el estrés que puede añadir» (Educación 2020, 2020, p.2).

En este contexto, la PC implementada por el MINEDUC (2020), indica que, como apoyo para la atención de la diversidad, adquiere especial relevancia el Decreto 83/2015 que tiene como propósito establecer las regulaciones para la adecuación curricular en el contexto de la educación inclusiva. Asimismo, la Agencia de Calidad de la Educación (2018), indica que los criterios señalados en dicho decreto, están basados en el Diseño Universal de Aprendizaje (DUA), el que busca responder a las diferencias de los estudiantes centrándose en los avances del aprendizaje y en las nuevas tecnologías.

Por otra parte, lo mencionado en el párrafo anterior, se complementa con lo descrito en las Bases Curriculares de Educación Física y Salud (MINEDUC, 2013), entre lo que se señala que:

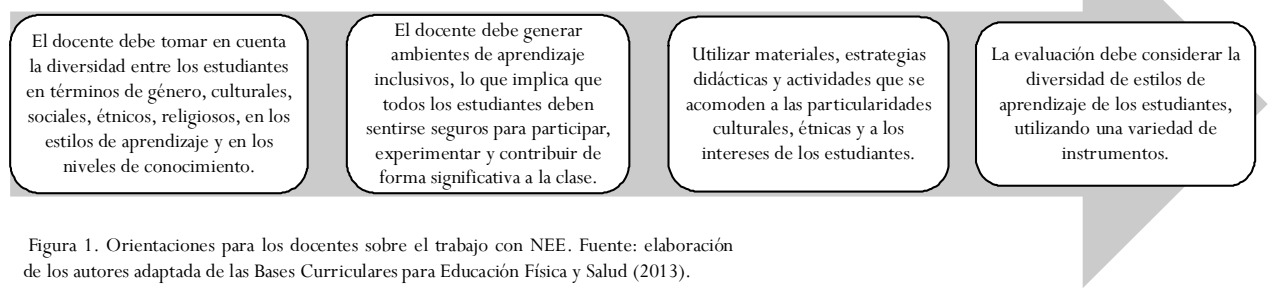

A partir de esto, es necesario que los elementos mencionados anteriormente se consideren en esta nueva realidad, para que de esta manera los estudiantes con NEE sean atendidos de una manera adecuada, para el logro de los aprendizajes priorizados, como también, los docentes brinden una ayuda significativa para los padres y apoderados, quienes, en este contexto, son guías y principal apoyo de los alumnos. En este estudio se estableció como objetivo conocer el nivel de atención de los estudiantes que presentan NEE en los centros de PSP en EF en tiempos de pandemia, 


\section{Metodología}

Se utilizó un enfoque cuantitativo, un diseño de investigación no experimental transversal con alcance descriptivo de tipo exploratorio. Con este procedimiento metodológico se logró la recolección de datos necesaria para obtener resultados satisfactorios relacionados con las variables indagadas y cuyos resultados obtenidos brindan una realidad específica a la que están sujetos, y exploratorio, ya que consiste en examinar un tema poco estudiado o que no se ha abordado antes (Sarduy, 2007; Hernández et al., 2010).

\section{Participantes}

La muestra fue no probabilística por conveniencia, con un total de 63 alumnos del de pedagogía en Educación Física, quienes realizaron su proceso de PSP durante el año, de los cuales un $87,3 \%$ desarrollaron este proceso en el primer semestre escolar y un 12,7\% la llevó a cabo en el segundo. El criterio de inclusión fue ser estudiante universitario de PSP de pedagogía en Educación Física. Los criterios de exclusión fueron: No haber terminado la encuesta o no haber trabajado con alumnos con NEE.

\section{Instrumentos}

Se les aplicó un cuestionario con 30 ítems. Las variables utilizadas estaban operacionalizadas en tres dimensiones o subvariables en relación a los datos sociodemográficos, datos relacionados a los centros educativos y a la PSP. Las técnicas y los instrumentos utilizados coinciden con el enfoque dado, en este caso se usó la encuesta como técnica y como instrumento, un cuestionario con 28 preguntas cerradas que responden a criterios de ponderación usando la escala de Likert, que va desde $1=$ totalmente en desacuerdo hasta $5=$ totalmente de acuerdo y 2 respuestas abiertas de las cuales los resultados siguieron un análisis cualitativo no considerados para este informe. Los datos fueron codificados y clasificados en categorías después de la simplificación, resumen y selección de la información, estas categorías respondieron a los siguientes criterios: a) Tipo de NEE presentadas por los estudiantes atendidos, b) modo de trabajo.

\section{Validez de contenido}

Se invitó a cinco profesores de doctorado con experiencia docente en la disciplina y enfocándose en su investigación en la línea de formación, conocimiento y desarrollo profesional en Educación Física (comité de expertos) para participar en el proceso de validación.

En la práctica, la validez de contenido se calculó sobre la base de en las respuestas de los expertos que evaluaron, respectivamente, si el lenguaje de cada ítem era claro, la dimensión teórica evaluada por los expertos tuvo como objetivo verificar la relevancia del elemento para cada una de las dimensiones teóricas del instrumento.

Para responder estas preguntas (claridad del lenguaje, relevancia práctica y dimensión teoría), se envió una hoja de cálculo a los especialistas escala Likert de uno a cinco puntos. Después de la evaluación por se proporcionaron sugerencias del comité de expertos para idoneidad que fueron analizados y probados en un estudio piloto con el objetivo de revisar y mejorar algunos términos técnicos, inicialmente propuestos.

\section{Procedimientos}

La encuesta se aplicó en estudiantes de Pedagogía en Educación Física de una universidad chilena vía correo electrónico durante los meses de agosto a septiembre 2020. En el correo se incluyó una breve descripción de la investigación con el objetivo y un enlace utilizando la herramienta Formulario de Google. Con relación al consentimiento informado, también fue online donde los sujetos debían leer y finalmente aceptar para poder contestar la encuesta, esto último siguiendo los criterios éticos expuesto por el tratado de Helsinki.

\section{Estadística}

Para el análisis de datos, se utilizó el programa estadístico IBM SPSS 18.0, el que permite trabajar con una gran cantidad de información, pero también permite el análisis de bases de datos, como es el caso. Para el tratamiento de la información, se creó una base de datos con los resultados obtenido, compuesto por 30 variables. Una vez obtenida esta base, un análisis descriptivo de los datos se llevó a cabo, basándose principalmente en el estudio de frecuencias y porcentajes,

\section{Resultados}

Este estudio se aplicó a los estudiantes que realizaron su PSP (79 individuos) durante el año académico 2020 , de los cuales un $87,3 \%$ desarrollaron este proceso en el primer semestre escolar y un 12,7\% la llevó a cabo en el segundo. De los datos obtenidos, relacionados específicamente a los centros educativos en los que efectuaron su PSP, se puede evidenciar cuál fue el nivel de compromiso que estos tuvieron para lograr la aten- 
ción de las NEE de sus estudiantes en contexto de pandemia. Para empezar, es necesario contextualizar la labor de los centros educativos en nuestro país y de qué manera se conforman, ante esto el MINEDUC (2016) indica que:

«Las escuelas, colegios y liceos son comunidades educativas lideradas por un director y un equipo directivo donde participan docentes, asistentes de la educación, estudiantes, madres, padres y apoderados, entre otros agentes. Estos actores escolares son los primeros responsables de los procesos educativos y de mejoramiento que ocurren en el sistema escolar y su misión primordial es educar integralmente a sus estudiantes» (p.17).

De lo anterior, los centros educativos que fueron parte de esta investigación pertenecen a diversas dependencias administrativas, de las que un 52,4\% corresponde a establecimientos municipales, un $42,9 \%$ a establecimientos particulares subvencionados, un 3,2\% a establecimientos particulares pagados y un 1,6\% a corporación de administración delegada. Asimismo, un $82,5 \%$ de los practicantes encuestados declaró que el establecimiento donde desarrolló su PSP cuenta con Programa de Integración Escolar (PIE), por lo tanto, admite a estudiantes con NEE, que según el MINEDUC (2016, p.9), es aquel que, «en razón de sus características o diferencias individuales o de su contexto (familiar, social, cultural u otras), enfrenta en la escuela algunas barreras que le dificultan o impiden avanzar en forma adecuada en su proceso educativo», observándose una estrecha relación entre la implementación del PIE con lo declarado en los Proyectos Educativos Institucionales (PEI), misión y visión de los establecimientos, toda vez que un $88,9 \%$ de los encuestados indica que estos apuntan hacia una educación de calidad con igualdad de oportunidades.

En relación con lo señalado, de la muestra total obtenida se logra apreciar en la figura 2 que un 98,4\% indica estar de acuerdo con que es un deber del centro educativo atender a los estudiantes con NEE en tiempos de pandemia, para lo cual, se precisa de un trabajo colaborativo entre todos los profesionales del establecimiento que según los practicantes encuestados $(66,1 \%)$, considera que esto es una labor fundamental para mejorar el proceso de atención a las NEE, lo que concuerda con lo mencionado en el informe de «La educación en tiempos de pandemia» publicado por CEPAL y la UNESCO (2020), donde se indica que en situaciones de emergencia las escuelas son un pilar fundamental para el apoyo socioemocional y continuidad del proceso de enseñanza-aprendizaje para los estudiantes, familias y comunidad educativa, debiendo responder a la diversidad de situaciones de cada uno de ellos y a sus necesidades de apoyo. Por otro lado, en lo dispuesto en el artículo 11 de la Ley 20.903 promulgada el año 2016, que «Crea el sistema de desarrollo profesional docente y modifica otras normas», se enfatiza «en la aplicación de técnicas colaborativas con otros docentes y profesionales, así como también el desarrollo y fortalecimiento de las competencias para la inclusión educativa» (p.3). De igual modo, la Mesa Social Covid-19 (2020), señala que las condiciones excepcionales en que nos encontramos demandan flexibilidad y aprendizaje continuo. Por lo tanto, es necesario que los equipos directivos reorganicen el modo de trabajo y generen condiciones para el trabajo colaborativo entre todos los miembros de la comunidad educativa.

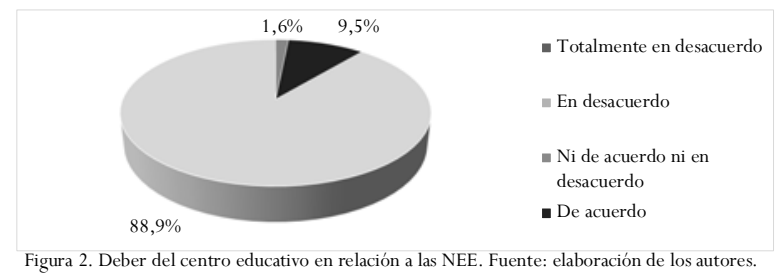

En el caso de la asignatura de Educación Física y Salud, gran parte de los docentes del área trabajó de manera colaborativa con todos los participantes del centro educativo, demostrando según los resultados evidenciados en esta encuesta $(57,2 \%)$, que hubo compromiso por parte de ellos para atender las NEE de todos sus estudiantes. No obstante, un 42,9\%, indica que hubo falta de compromiso por parte de los docentes de los establecimientos de PSP, debiendo reforzar este aspecto puesto que, según lo mencionado por Juanes et al. (2020) y Muñoz, Smith \& Matos, 2020, una de las prácticas más importantes que se deben considerar al momento de planificar y ejecutar una clase virtual es el compromiso o fidelidad, aspectos que provocarán elevar los niveles de compromiso, participación e interacción, mejorando los resultados como la integración de herramientas tecnológicas de uso cotidiano, implementación de un modelo de acompañamiento para docentes y estudiantes y crear contenidos de calidad, entre otros.

Lo anterior se relaciona con la preocupación demostrada por parte de los centros educativos por lograr la inclusión de todos sus estudiantes, puesto que como se puede apreciar en la figura 3, un 65,1\% indica que sí hubo preocupación, sin embargo y no menos importante un $34,9 \%$ de la muestra, menciona que este aspecto 
tuvo carencias, además, 53,9\% de los practicantes señalan que su centro educativo le otorga importancia a las NEE, por el contrario un $46,1 \%$ de ellos manifiesta que esto no fue así, esto porque de acuerdo a lo indicado por Murillo y Duk (2020), en el contexto de la emergencia sanitaria, los estudiantes que presentan alguna discapacidad se han visto confinados a sus casas, siendo en general olvidados por las medidas paliativas que los sistemas educativos han tomado para suavizar esta situación, por ende, la educación a distancia se convierte para muchos en un imposible. Ante esto, REDI (2020), destaca la importancia de garantizar el acceso a los materiales educativos, en formatos accesibles, con las adaptaciones requeridas para los y las estudiantes con discapacidad que se encuentran en esta situación.

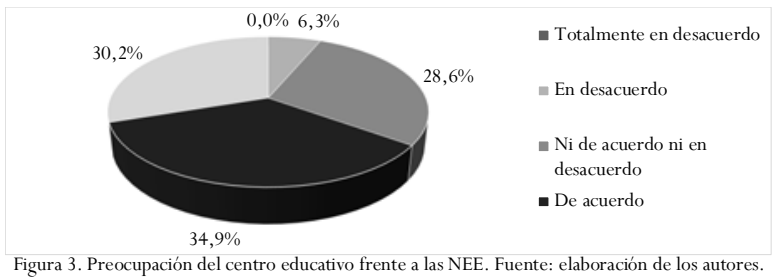

Por último, se hace imprescindible el trabajo que realiza el equipo que conforma el PIE, que según el MINEDUC (2016), es una estrategia educativa con enfoque inclusivo que se implementó en los establecimientos educacionales regulares con el propósito de favorecer la participación y el logro de los objetivos de aprendizaje de todos los estudiantes, aportando recursos y equiparando las oportunidades educativas, especialmente para aquellos que presentan mayores necesidades de apoyo para progresar en sus aprendizajes. Es por esto que, como se puede observar en la figura 4, el 97\% de los encuestados considera fundamental el trabajo del equipo PIE en respuesta a las NEE del estudiante en tiempos de pandemia, lo que se respalda por lo mencionado en el informe Educar en tiempos de pandemia publicado por la Organización Educación 2020 (2020), el trabajo colaborativo con los equipos PIE es un elemento clave para el crecimiento profesional y el bienestar de toda la comunidad educativa, pues fomenta el desarrollo de una cultura escolar que valore la diversidad, puesto que el equipo PIE junto con el equipo directivo, poseen las herramientas adecuadas para liderar la

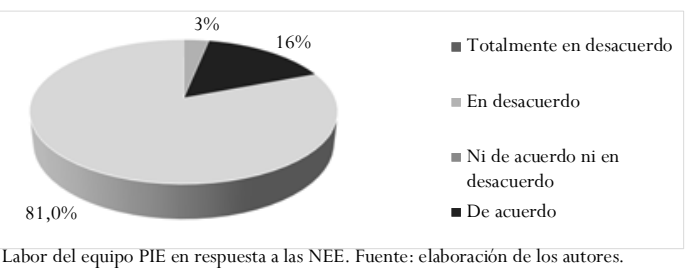

reflexión que permite colaborar de forma efectiva en este proceso en beneficio del bienestar emocional de docentes y estudiantes y los progresos a nivel de aprendizaje para su trayectoria formativa.

La actitud de la familia como factor clave en el progreso de los aprendizajes de los estudiantes con NEE en tiempos de pandemia es algo que también se ha evidenciado en los resultados obtenidos, como se observa en la figura 5, el 100\% de los encuestados considera que esto influye en el proceso de enseñanza-aprendizaje de los alumnos con NEE, antecedentes que concuerdan con lo señalado por Mesa Social Covid-19 (2020), donde se indica que es necesario que el adulto significativo para el niño sea capaz de fomentar un estado de ánimo optimista frente a la tarea, de tal manera que signifique las experiencias de error como remontables y que promueva un ambiente socioemocional que aliente la práctica y el esfuerzo. Así también, Educación 2020 (2020), señala que los adultos responsables deben promover las condiciones para que el educando aprenda, siendo estas la generación de un ambiente adecuado, materiales necesarios, fomento de la asistencia a clases y, en la medida de sus posibilidades, apoyo en el trabajo pedagógico.

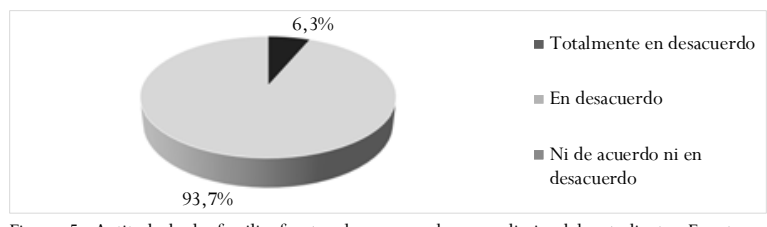

Figura 5. Actitud de la familia frente al proceso de aprendizaje del estudiante. Fuente: elaboración de los autores.

En la actualidad, la PSP ha debido adaptarse a la nueva realidad surgida producto de la pandemia del Covid19, transformándose de esta manera, la educación virtual, en la mejor herramienta para desarrollar el proceso de enseñanza-aprendizaje durante este año y así, a quienes les correspondía culminar su formación académica, pudieran realizarlo en la medida y forma que esta pandemia lo permitió. En el caso de la carrera de Pedagogía en Educación Física de la Universidad Católica del Maule, se llevó a cabo un módulo de carácter teóricopráctico cuyo propósito consideraba poner en práctica las competencias adquiridas en el transcurso del itinerario formativo, consistente en que el estudiante asista a un establecimiento educacional de manera virtual durante 11 horas semanales, en donde desarrolle su rol de profesor practicante y funciones de profesor jefe.

Relacionado con lo anterior, en el contexto de la PSP, los practicantes debieron desarrollar el proceso de enseñanza-aprendizaje de sus estudiantes de la mejor manera posible, incluyendo la atención de aquellos alum- 
nos que presentan alguna NEE. Dentro de los resultados obtenidos, un alto porcentaje atendió a estudiantes con NEE asociadas a diversas discapacidades (figura 6), entre las cuales se encuentra un $31,7 \%$ a estudiantes con discapacidad motora, un $12,7 \%$ a estudiantes con discapacidad visual, un $1,6 \%$ a estudiantes con discapacidad auditiva y un $22,2 \%$ a estudiantes con discapacidad intelectual o cognitiva, siendo un total de $68,2 \%$ de estudiantes que poseen una NEE. Por otro lado, la modalidad de trabajo que más utilizaron los practicantes fue la entrega de material escrito (guías, ppt, etc.) y material audiovisual (cápsulas de videos, cápsulas informativas, etc.) y, en menor medida, la realización de clases online vía plataformas virtuales (Meet, Zoom, Teams, entre otras).

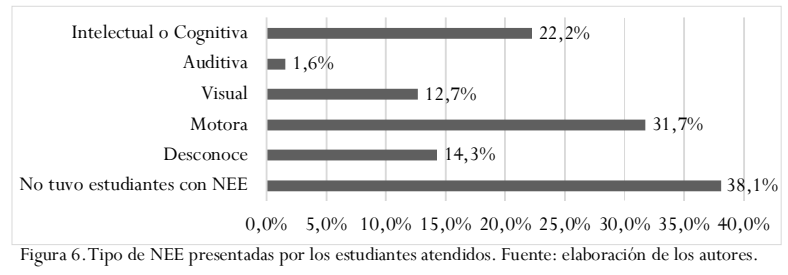

De acuerdo a los datos anteriormente descritos, se logra apreciar que la atención a la diversidad es un elemento a considerar por los encuestados dentro de la PSP, siendo un 95,2\% quienes concuerdan con esta afirmación, ante lo cual Educación 2020 (2020), señala que es necesario asegurar el acceso, realizar monitoreo y apoyos personalizados a los estudiantes que reciben subvención del PIE y los que no, por lo que se requiere realizar las adecuaciones pertinentes y buscar diversas herramientas para dar respuesta a las necesidades de cada uno de ellos. Es por esto que los practicantes $(66,6 \%)$ mencionan que las actividades planificadas estaban orientadas a lograr la inclusión de todos los estudiantes. Sin embargo, a pesar de lo anterior, en la figura 7, se observa que un alto porcentaje $(66,7 \%)$ indica que quienes poseen una NEE no reciben una adecuada atención bajo esta nueva modalidad de educación virtual, lo que se debe según el MINEDUC (2020), a que en la educación a distancia se han revelado importantes brechas en cuanto a la heterogeneidad de capacidades y recursos disponibles por quienes imparten la enseñanza como por aquellos que necesitan acceder a ella, puesto que por medio

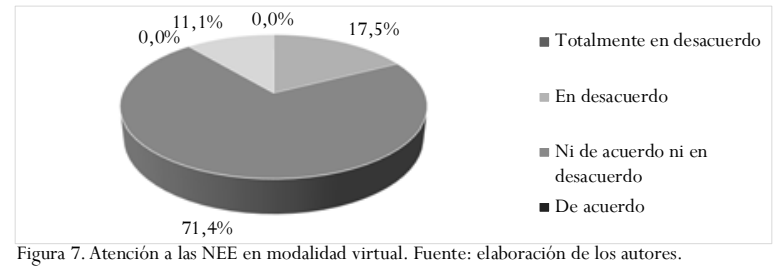

de una comparación entre los establecimientos de mayor vulnerabilidad con los de menor vulnerabilidad, se ha evidenciado que los primeros poseen una cobertura de sólo el 27\% de sus estudiantes, en cambio los segundos, alcanzan un $89 \%$.

Por consiguiente y de acuerdo a la experiencia de la muestra obtenida, en la figura 8, un 73\% de ella considera que no es posible atender las NEE de todos los estudiantes por medio de la virtualidad, ya que, en concordancia con lo mencionado por Álvarez y Figares (2020), a pesar de los esfuerzos por seguir con las actividades de apoyo a los estudiantes con NEE realizados por los establecimientos y quienes lo componen, se advierte que estas intervenciones, de por sí complejas, se convierten en menos efectivas en comparación a lo habitual, debido a la dificultad que existe para observar directamente el desempeño de los estudiantes y la débil capacidad para retroalimentar de manera adecuada. Además, un 49,2\% indica que, en el caso de la asignatura de Educación Física y Salud, se logró la consecución de aprendizajes de los estudiantes con NEE en tiempos de pandemia, por el contrario, un 50,8\% se encuentra en desacuerdo con lo anterior. A pesar de esto Gil (2020), menciona que posiblemente esta ha sido la mejor oportunidad para trabajar el desarrollo de la autonomía y la participación familiar, aspectos imprescindibles para llevar a cabo una EF de calidad y de éxito.

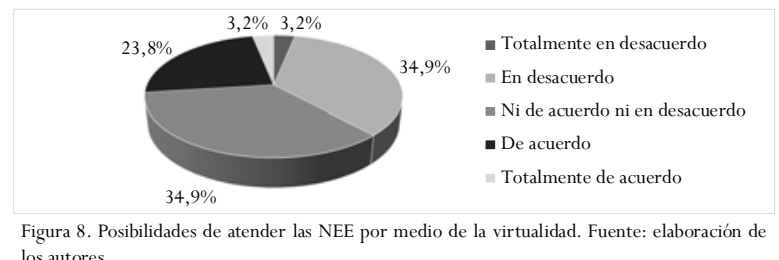
los autores.

Por otro lado, en cuanto a la formación inicial docente, si bien en la figura 9 se aprecia que un 50,8\% menciona que adquirió suficientes conocimientos sobre atención a las NEE durante la carrera, un porcentaje no menor $(49,2 \%)$ y por ende preocupante, se encuentra en desacuerdo con lo anterior. En consecuencia, un $52,3 \%$ se considera suficientemente preparado para abordarlas pedagógicamente, por el contrario, un 47,6\% señala que no se encuentran preparados para enfrentar este desafío. Estos antecedentes concuerdan con algunas publicaciones (Symeonidou, 2017; Campos et al., 2020; Solís \& Borja, 2021), en donde se expone que la mayoría del estudiantado indica no haber recibido suficiente formación en las temáticas de diversidad, integración escolar y necesidades educativas especiales, por lo tanto, no se siente preparado para la inclusión. De igual modo, según un estudio realizado por Hinojosa et al. 
(2020), se evidencia una baja proporción, tanto de universidades estatales como privadas, en el desarrollo de competencias profesionales vinculadas con el respeto a la diversidad como parte de una educación y desarrollo integral.

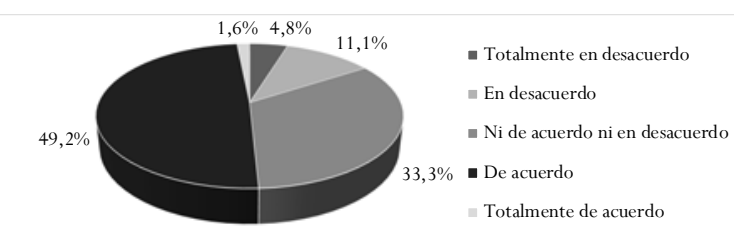

Figura 9. Adquisición de conocimientos durante la carrera. Fuente: elaboración de los autores.

Finalmente, es importante destacar que la atención a la diversidad puede llevarse a cabo en todas y cada una de las áreas que componen el currículum escolar priorizado, puesto que los mismos encuestados $(77,8 \%)$ indican que esto es posible. En primer lugar, porque el MINEDUC (2020) ha creado una propuesta curricular que prioriza los objetivos de todas las asignaturas, excepto las de Orientación y Tecnología, en segundo lugar, porque dicha priorización se sustenta en tres principios básicos: seguridad, flexibilidad y equidad, sumándose el principio que define la educación de calidad desde la atención efectiva a la diversidad.

\section{Discusión y conclusiones}

Esta investigación fue aplicada a estudiantes que realizaron su PSP durante el año 2020, quienes desarrollaron su proceso en diferentes centros educativos bajo la modalidad virtual, situados entre la sexta y séptima región de Chile. El objetivo establecido fue conocer el nivel de atención de los estudiantes que presentan NEE en los centros de Práctica de Síntesis Profesional (PSP) en EF en tiempos de pandemia a través de la percepción de los practicantes.

Cada centro educativo posee diferentes criterios en los cuales basa el tipo de educación que entrega, con el propósito de que esta sea de calidad. Entre estos lineamientos se encuentran los deberes del establecimiento, siendo el principal otorgar educación e igualdad de oportunidades a los estudiantes, adecuándose a los diversos requerimientos del contexto, ya sea de manera presencial o virtual.

En relación a lo anterior, por medio de este estudio, se obtuvo que un $98,4 \%$ de los practicantes concuerda con que es un deber del centro educativo atender a los estudiantes con NEE en tiempos de pandemia, lo que debería ser directamente proporcional con la preocupación que demuestra el establecimiento por lograr un adecuado nivel de atención de estos alumnos. Sin embargo, uno de los hallazgos es que si bien existió esa preocupación en muchas de las escuelas estudiadas, se observó que hubo carencias en este aspecto, lo que podría deberse a lo señalado por Murillo y Duk (2020), quienes indican que en el contexto de alerta sanitaria, los estudiantes que presentan alguna discapacidad, en general, han sido olvidados por las medidas que los sistemas educativos han dispuesto para enfrentar esta situación, lo que ha provocado que la educación a distancia se convirtiera en un trabajo difícil de sobrellevar. En este sentido, la atención a las NEE se ha visto altamente dificultada por la adversidad del contexto actual, provocando que en muchos casos el trabajo realizado sea de manera homogénea y no heterogénea.

Por otro lado, un factor importante que se debe considerar a la hora de atender las NEE, es la labor fundamental que realiza el equipo PIE en respuesta a las necesidades educativas de los estudiantes, puesto que los mismos encuestados indican que esto es de suma relevancia para el logro del proceso de enseñanza-aprendizaje. Como también y más aún en el contexto de pandemia en el que nos encontramos, otro elemento que influye significativamente en la adquisición de los aprendizajes de los alumnos, es la actitud que demuestra la familia. En efecto, Educación 2020 (2020) en primera instancia, en primera instancia, indica que para que se desarrolle una cultura escolar que valore la diversidad, es clave que se genere un trabajo colaborativo con los equipos PIE, y en segunda instancia, menciona que es necesario que los adultos responsables promuevan las condiciones para que el educando pueda aprender. Ambos factores, son imprescindibles para que se logre un proceso de enseñanza-aprendizaje efectivo en los estudiantes, especialmente de aquellos que presentan alguna NEE, es por esto, que es fundamental el trabajo colaborativo entre todos los miembros de la comunidad educativa.

Otro de los hallazgos obtenidos, es que no se percibe una atención adecuada en modalidad virtual de aquellos estudiantes que presentan NEE, puesto que no hubo una consecución de los aprendizajes esperados, por lo tanto, se puede inferir que no es posible atender las NEE en una modalidad virtual, lo que concuerda con lo mencionado por Álvarez y Figares (2020), , quienes señalan que, debido a la dificultad para observar directamente el desempeño de los estudiantes, las intervenciones se convierten en menos efectivas. Esto se produce, ya que, al enfrentarse a una nueva realidad, se ha generado un momento de incertidumbre para los pro- 
fesionales de la educación, quienes, ante la difícil tarea de continuar con el proceso educativo a distancia, han debido implementar diversas estrategias de enseñanza de las cuales no poseían experiencias previas y que, por lo tanto, no permiten el logro de los aprendizajes esperados.

Por último, un resultado de alto impacto que se halló en este estudio, es que si bien un 50,8\% de los practicantes a los que se les aplicó la encuesta indica que adquirió suficientes conocimientos en el área de las NEE, un porcentaje no menor $(49,2 \%)$ se encuentra en desacuerdo con ello. En consecuencia y debido a lo anterior, es que un número importante de los practicantes, no se siente preparado para afrontar el desafío de atención a las NEE, tanto de manera presencial como virtual. Ante esto, Symeonidou (2017), señala que gran parte del estudiantado no se siente preparado para la inclusión, debido a la falta de preparación en esta materia. Asimismo, Hinojosa at al. (2020), mencionan una deficiencia que existe en generar competencias asociadas a la diversidad por parte de las universidades, tanto estatales como privadas. Es decir, parte de esta falta de preparación que dicen tener los practicantes encuestados, se debe a la baja formación inicial recibida sobre competencias vinculadas a la atención de las NEE e inclusión en general.

Para concluir, se determina que existió atención en muchos de los centros educativos estudiados, algunos de ellos todavía requieren mejorar en este aspecto para atender a sus estudiantes con NEE, apreciando en estos últimos, un nivel descendido, lo que deja en evidencia que en el contexto de pandemia el abordaje de las NEE se ha dejado en segundo plano, puesto que se le ha dado mayor énfasis al cumplimiento del currículum priorizado a través de una modalidad de trabajo homogénea. También es importante y más aún en estos tiempos, que los establecimientos involucren más a las familias en el proceso de enseñanza-aprendizaje de sus hijos, además de que los docentes se preocupen en especializarse en temas de inclusión educativa y atención a las NEE, si bien, existe el decreto 83 que busca diversificar la enseñanza, aún queda mucho camino por recorrer y aprender en este sentido.

Por otro lado, esta investigación demostró que existe una carencia en la formación inicial recibida en el área de las NEE, concordando con el estudio de Flores \& Maureira (2020), lo que no permite abordarlas al cien por ciento, tanto de manera presencial como virtual. Por lo tanto, es fundamental que las universidades se preocupen de entregar una formación apropiada res- pecto a la atención de las NEE y la inclusión, siendo necesario que esta área se trabaje de manera transversal durante la formación inicial de los futuros docentes. Por consiguiente, se considera imprescindible que el docente a partir de su formación y su propia motivación, se preocupe por buscar estrategias acordes a las necesidades de sus estudiantes, más aún, de aquellos que poseen una NEE puesto que, en nuestra área, si se aplica un mal método, puede generar daños en los estudiantes.

\section{Referencias}

Agencia de Calidad de la Educación. (2018). Los desafíos de educar para la inclusión y la diversidad. División de Información a la comunidad. Recuperado de https:/ /archivos.agenciaeducacion.cl/IDH_web.pdf

Almonacid-Fierro, A., Souza de Carvalho, R., CastilloRetamal, F. \& Almonacid, M. (2021). The Practicum in Times of Covid-19: Knowledge Developed by Future Physical Education Teachers in Virtual Modality. International Journal of Learning, Teaching and Educational Research, 20(3), 68-83. https://doi.org/ 10.26803/ijlter.20.3.5

Álvarez-Hevia, D., \& Figares, J. (2020). Retos Educativos durante el Confinamiento: La Experiencia con Alumnos con Necesidades Educativas Especiales. Revista Internacional de Educación para la Justicia Social, 9(3), 1-11. https://revistas.uam.es/riejs/article/ view/12229

Arriagada, P. (2020). Pandemia Covid-19: Educación a Distancia. O las Distancias en la Educación. Revista Internacional de Educación para la Justicia Social, 9(3), 1-3. https://revistas.uam.es/riejs/article/view/ 12396

Campos Granell, J., Llopis Goig, R., Gimeno Raga, M., \& Maher, A. (2020). Percepción de competencia para la atención de alumnos con necesidades educativas especiales en Educación Física: la voz de estudiantes universitarios de España y Reino Unido (Perceived competence to teach students with special educational needs in Physic. Retos, (39), 372-378. https://doi.org/10.47197/retos.v0i39.79498

Castillo-Retamal, F., Cárcamo Garrido, B., Aravena Calderón, H., Valenzuela Zakuda, A., Pérez Farías, T., Medel Tapia, C., \& Quezada Alacaino, J. (2021). Necesidades Educativas Especiales y Educación Física: un análisis desde la propuesta curricular ministerial de Chile (Special Educational Needs and Physical Education: an analysis from the Chilean ministerial 
curricular proposal). Retos, 42, 56-65. https:// doi.org/10.47197/retos.v42i0.86977

Castillo, F., Andrade, S., Arias, N., Cabrales, F. \& Díaz, J. (2015). Necesidades educativas especiales y Educación Física: Las actividades en la naturaleza como instrumento socializador. Revista Licere, 18(4), 71-93. https://doi.org/10.35699/1981-3171.2015.1157

CEPAL-UNESCO. (2020). La educación en tiempos de la pandemia de COVID-19. Naciones Unidas. https:// unesdoc.unesco.org/ark:/4 $8223 /$ pf0000374075?locale $=$ es

Colegio de Profesoras y Profesores de Chile. (2020). Orientaciones para el trabajo pedagógico en tiempos de pandemia. Departamento de Educación. http:// revistadocencia.cl/web/images/orientaciones/ Orientaciones_1.pdf

Educación 2020. (2020). Educar en tiempos de pandemia. Parte 4: Recomendaciones para gestionar la diversidad. Santiago de Chile. http://educacion2020.cl/wpcontent/uploads/2020/06/ Orientaciones_documento4.pdf

Emanuel, E. J., Persad, G., Upshur, R., Thome, B., Parker, M., Glickman, A., Zhang, C., Boyle, C., Smith, M., \& Phillips, J. P. (2020). Fair allocation of scarce medical resources in the time of Covid-19. The New England Journal of Medicine, 322(21), 20492055. https://doi.org/10.1056/NEJMsb2005114

Flores-Ferro, E., Maureira-Cid, F., Hadweh-Briceño, M., Gutiérrez-Duarte, S., Silva-Salse, Á., PeñaTroncoso, S... Lagos-Olivos, C. (2021). Nivel de satisfacción de las clases online por parte de los estudiantes de Educación Física de Chile en tiempos de pandemia (Level of satisfaction of online classes by students of Physical Education of Chile in times of pandemic). Retos, 41, 123-130. https://doi.org/ 10.47197/retos.v0i41.82907

Flores-Ferro, E. \& Maureira-Cid, F. (2020). Formación pedagógica en la carrera de educación física: Falta de conocimientos para un profesional del siglo XXI. EmásF: revista digital de educación física, 62, 118 126. http://emasf2.webcindario.com/ EmasF_62.pdf

Gil-Espinosa, F. (2020). La covid-19: una carrera de fondo para avanzar en educación física. Journal of Physical Education and Human Movement, 2(2), 1-5. https:// doi.org/10.24310/JPEHMjpehmjpehm.v2i29849

Hernández Sampieri, R., Fernández, C. \& Baptista, M. (2010). Metodología de la investigación (Quinta ed.). The McGraw-Hill. https://www.esup.edu.pe/desc a r gas / d e p_investiga cion/

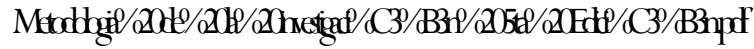

Hinojosa Torres, C., Hurtado Guerrero, M., \& Magnere Ávalos, P. (2020). Profesores noveles de educación física: percepciones sobre su formación docente en base al desempeño en el sistema escolar (Novel physical education teachers: perceptions on their teaching training based on performance in the school system). Retos, 38(38), 396-405. https://doi.org/ $10.47197 /$ retos.v38i38.75212

Holz, M. (2018). Datos de la modalidad de Educación Especial en Chile, año 2018. Biblioteca del Congreso Nacional de Chile. https://obtienearchivo.bcn.cl/ obtienearchivo?id=repositorio/10221/26781/2/ BCN_datos_de_EE_y_estudiantes_con_NEE_Final.pdf Jiménez, F., \& Montecinos, C. (2018). Diversidad, modelos de gestión y formación inicial docente: desafíos formativos desde una perspectiva de justicia social. Revista Brasileira de Educação, 23, 1-21. https:// doi.org/10.1590/s1413-24782018230005.

Juanes, B., Munévar, O., \& Cándelo, H. (2020). La virtualidad en la educación, aspectos claves para la continuidad de la enseñanza en tiempos de pandemia. Revista Conrado, 16(76), 448-452. https:// conrado.ucf.edu.cu/index.php/conrado/article/ view $/ 1510$

Ley $\mathrm{N}^{\circ}$ 20370. Diario Oficial de la República de Chile, Santiago, Chile, 12 de septiembre de 2009. Biblioteca del congreso nacional. https://especial.mineduc.cl/wpcontent/uploads/sites / 31 / 2018/04 / LEY20370_12-SEP-2009.pdf

Ley 20.903. Crea el sistema de desarrollo profesional docente y modifica otras normas. Santiago, Chile, 1 de abril de 2016. Biblioteca del congreso nacional. https:// www.bcn.cl/leychile/navegar?idNorma $=1087343$

Mesa Social Covid-19. (2020). Didácticas para la proximidad: aprendiendo en tiempos de crisis. Propuestas Educación Mesa Social Covid-19, 15. https:// www.lidereseducativos.cl/recursos/informedidacticas-para-la-proximidad-aprendiendo-en-tiempos-de-crisis/

MINEDUC. (2013). Bases Curriculares Educación Física y Salud. Santiago, Chile. https:// www.curriculumnacional.cl/portal/Educacion-General/Educacion-fisica-y-salud/

MINEDUC. (2015). Diversificación de la Enseñanza. Decreto $N^{\circ} 83 / 2015$. Santiago. Santiago: MINEDUC. https://especial.mineduc.dl/wp-content/uploads/ sites/31/2016/08/Decreto-83-2015.pdf

MINEDUC. (2016). Plan de Aseguramiento de la Calidad Escolar 2016-2019 (SAC). Sistema de Aseguramiento 
de la Calidad de la Educación, 5-40. de https:// bibliotecadigital.mineduc.cl/bitstream/handle/ $20.500 .12365 / 442 / \mathrm{M} \mathrm{O} \mathrm{N} \mathrm{O} \mathrm{-}$ 369.pdf?sequence $=1$ \&isAllowed $=\mathrm{y}$

MINEDUC. (2016). Programa de Integración Escolar PIE. Ley de Inclusión 20. 845. Manual de apoyo a la Inclusión Escolar en el marco de la Reforma Educacional. Santiago: MINEDUC. https://especial.mineduc.cl/wpcontent/uploads/sites/31/2017/12/Manual-PIE.pdf

MINEDUC. (2020). Estudiar en tiempos de coronavirus. Revista de Educación, 389, 7-11. http:// www.revistadeeducacion.cl/revista_pdf/ reveduc_389/files/assets/common/downloads/ REVEDUC\%20389.pdf

MINEDUC. (2020). Fundamentación Priorización Curricular Covid-19. Unidad de Currículum y Evaluación. Santiago: MINEDUC. https:// www.curriculumnacional.cl/portal/Secciones/ Curriculum-transitorio/178042: PriorizacionCurricular

MINEDUC. (2020). Impacto del COVID-19 en los resultados de aprendizaje y escolaridad en Chile. Santiago: Centro de Estudios MINEDUC. https:// www.mineduc.cl/impacto-del-covid-19-en-los-resultados-de-aprendizaje-en-chile/

MINEDUC. (2020). Orientación al sistema escolar en contexto de COVID-19. Santiago: División Educación General. https://www.mineduc.cl/wpcontent/uploads/sites/19/2020/03/ OrientacionesContextoCOVID19_2703.pdf

MINEDUC. (2020). Priorización curricular covid-19 educación física y salud. Santiago: Ministerio de Educación de Chile.https://www.curriculumnacional.cl/ portal/Secciones / Curriculum-transitorio/ 178042:Priorizacion-Curricular

MINEDUC. (26 de junio de 2020). Resolución Exenta $\mathrm{N}^{\circ}$ 2765. Implementacion de la Priorizacion curricular en forma remota y presencial. Santiago: Ministerio de Educación de Chile. https:// www.curriculumnacional.cl/portal/DocumentosCurriculares / Bases-curriculares / 209381:Resolucion-Exenta-N-2765-Implementacion-de-la-Priorizacio-n-curricular-en-forma-remota-ypresencial

MINSAL. (29 de abril de 2020). Resolución Exenta ${ }^{\circ}$ 322. Dispone medidas sanitarias que indica por brote de Covid-19. Santiago de Chile: Biblioteca del Congreso Nacional de Chile. https:/ /www.bcn.cl/ leychile $/$ navegar?idNorma $=1144710$

Molina, Y. (2015). Necesidades educativas especiales, elementos para una propuesta de inclusión educativa a través de la investigación acción participativa. El caso de la Escuela México. Estudios Pedagógicos, 41(Especial), 147-167. http://dx.doi.org/10.4067/S071807052015000300010

Muñoz Moreno, A., Smith, E., \& Matos Duarte, M. (2020). Una Experiencia de Inclusión Educativa en el Aula de Expresión Corporal con Alumnado Universitario (An Educational Inclusion Experience in a Body Expression course with undergraduate students). Retos, 37, 702-705. https://doi.org/ $10.47197 /$ retos.v37i37.68438

Murillo, J., \& Duk, C. (2020). El Covid-19 y las Brechas Educativas. Revista Latinoamericana de Educación Inclusiva, 14(1), 11-13. http://dx.doi.org/10.4067/ S0718-73782020000100011

Navarrete, M. (2019). Inclusión del Siglo XXI: reflexiones sobre la Educación Inclusiva en Chile y Latinoamérica. Polyphônia. Revista de Educación Inclusiva, 3(2), 175-176. https://revista.celei.cl/ index.php/PREI/article/view/93

REDI. (15 de 04 de 2020). Red por los derechos de las personas con discapacidad (REDI). Obtenido de Situación de las personas con discapacidad frente a la pandemia de COVID-19. http://www.redi.org.ar/ Prensa/Comunicados/2020/20-04-15_situacion-delas-personas-con-discapacidad-frente-a-la-pandemiade-Covid-19.pdf

Sarduy, Y. (2007). El análisis de información y las investigaciones cuantitativa y cualitativa. Revista Cubana de Salud pública, 33(3), 1-11. http://scielo.sld.cu/ scielo.php?script $=$ sci_arttext\&pid $=$ S086434662007000300020

Solís García, P., \& Borja González, V. (2021). Actitudes del profesorado de Educación Física hacia la inclusión de alumnos con discapacidad (Physical Education teachers' attitudes towards the inclusion of students with disabilities). Retos, 39, 7-12. https://doi.org/ 10.47197/retos.v0i39.77841

Symeonidou, S. (2017). Initial teacher education for inclusion: a review of the literature. Disability \& Society, 32(3), 401-422. https: / / doi.org/10.1080/ 09687599.2017 .1298992

Wallenius, B. (2020). Enseñanza en valores a través de la educación física (covid-19). [Trabajo de fin de grado, Universidad de La Laguna]. https://riull.ull.es/ xmlui/bitstream/handle/915/20822/

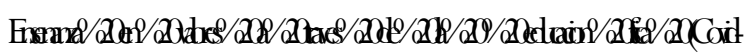
19).pdf?sequence $=1$ 